\title{
A Study of the Motor Unit Action Potential by Means of Computer Simulation
}

\author{
P. A. M. Griep, K. L. Boon, and D. F. Stegeman \\ Bio-Information Group, Department of Electrical Engineering, Twente University of Technology, Enschede, The Netherlands
}

\begin{abstract}
In order to study the motor unit action potential a computer simulation model was developed. It is based on the superposition of single muscle fibre potentials of the fibres belonging to the motor unit. The parameters which characterize each fibre (spatial position, diameter, and a dispersion of arrival time of the potential at the electrode) are chosen from statistical distributions which can be derived from anatomical and physiological data. The electrode type, position and dimensions can be specified. Simulated motor unit action potentials are presented in the time and frequency domain. The simulation results refer to (1) the influence of the electrode position and dimensions with respect to the motor unit territory, (2) the meaning of this model for the study of pathological phenomena, (3) the variability of some parameters characterizing the motor unit, (4) the selectivity of uni- and bipolar electrodes and finally (5) the influence of the geometrical situation of the motor end-plates within the muscle, on the shape of motor unit action potentials.
\end{abstract}

\section{Introduction}

The motor unit action potential (muap; plural muaps) of a certain motor unit can be considered as a summation of fibre potentials of all muscle fibres belonging to that motor unit. In Figure 1a a schematic drawing of one motor unit in a muscle is given. The shape of a muap and its power spectrum depend on a number of factors. The most important are: scattering and diameter of the muscle fibres of one motor unit, position and dimension of the electrode, conduction velocity, and the time dispersion meaning that there is a spread in the arrival time of the fibre potentials at the electrode. This dispersion is mainly caused by the different conduction velocities and lengths of the nerve twigs and the different positions of the motor end-plates. All these factors together form an obstacle for analytic calculation of possible muap shapes. However by means of simplified models some mathematical investigations have been carried out by George (1970) and very extensively by Lindström et al. (1976).

These models are interesting, especially in order to see how certain measurable parameters may reveal information about the motor unit, but their meaning with respect to a number of specific muap shapes is limited. The present study was undertaken to explore possible muap shapes and their relation to important anatomical and physiological parameters by means of computer simulation. This simulation was based on the fact that with a computer it is feasible to calculate the potential contribution of each muscle fibre of a motor unit separately. The potential contribution of a muscle fibre as used in the model, is described by Lorente de Nó (1947) and Rosenfalck (1969). Ekstedt and Stålberg (1973) described the influence of the size of the leading-off surface.

\section{Methods}

\subsection{General Principle}

The muap is calculated basically as a summation of separate muscle fibre potentials according to the superposition principle. The spatial position, the diameter of each fibre and the dispersion in arrival time of each fibre potential at the electrode can be chosen according to probability density functions (pdf's) or if preferred in a deterministic way. These pdf's depend of course on the muscle being studied and can be estimated from anatomical and physiological data. The proposed model is very well suited for general use because it can be adjusted in a simple way to anatomical and physiological data. In this study unipolar or bipolar "registrations", based on data of the $m$. soleus of the rat have been calculated. The potential of the indifferent electrode, when unipolar "registrations" were made, was taken as zero: in reality this means 


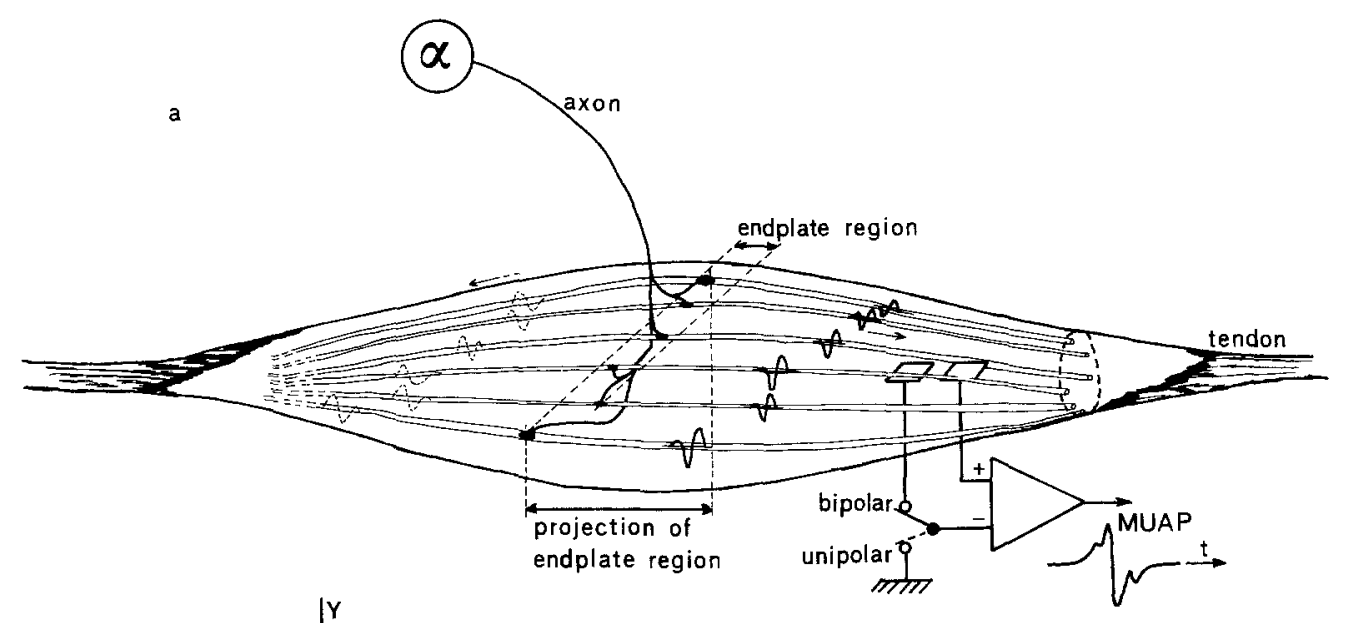

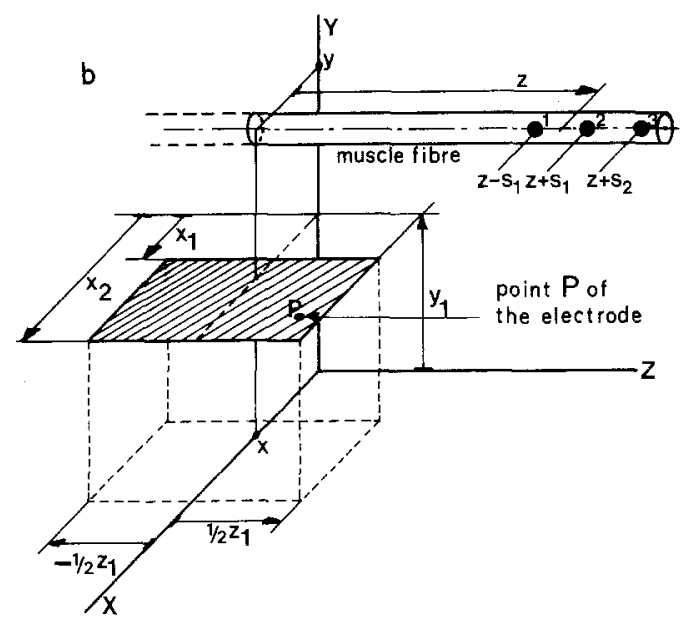

that such an electrode was thought to be placed in an electrical passive region.

The model was programmed in Fortran and can be executed on a minicomputer ${ }^{1}$. In Fig. 2 a typical example of a simulation result is shown. In the upper right quadrant the fibre positions and the electrode position and dimensions are drawn. The electrode position is intraterritorial in this example. In the upper left quadrant the dispersion, the distance to the electrode-centre and the fibre diameter for each fibre are drawn. The calculated muap is plotted in the lower left quadrant. The power spectrum is shown in the lower right quadrant since it is an important characteristic of the motor unit (Lindström, 1974; Leifer and Pinelli, 1976). In this study it will be shown how the coordinates of the low frequency maximum are related to specific anatomical and physiological data of the motor unit.

1 A detailed description and program listing is given in an internal technical report MUAP8 which can be obtained on request
Fig. 1a and b. Schematic drawing of one motor unit in a muscle and the coordinate system used. a Of the motor unit only 6 muscle fibres are drawn. Notice that the endplate region resembles to a skew zone with a certain thickness. The muscle fibre potentials which are travelling to the right direction can be "measured" in an unipolar or bipolar way. b A geometrical representation of one muscle fibre and the electrode. The coordinates $x_{1}, x_{2}$ and $z_{1}$ refer to the dimensions of the electrode. The coordinates $z-s_{1}, z+s_{1}$ and $z+s_{2}$ refer to the poles of the tripole [Eq. (1)]

\subsection{Calculation of a Fibre Potential}

The muscle tissue was considered as an homogeneous, anisotropic, unlimited volume conductor. The potential field in a volume conductor is subjected to the Laplace equation (Rosenfalck, 1969). Using the assumption that the potential field of one fibre is caused by three moving point-shaped current sources, this field can easily be described by the tripole model of Rosenfalck. It was assumed that the measuring electrode was placed far enough from the end-plate region so that the end-plate potentials and fibre potentials originating from parts of the fibre on the other side of the end-plate can be neglected. The potential contribution $\varphi_{p}^{\prime}$ for an isotropic medium of each muscle fibre with coordinates $(x, y)$ to a point $p$ (see Fig. 1b) with coordinates $\left(x_{i}, y_{i}, z_{i}\right)$ of the electrode was calculated according to the tripole model mentioned above. It was assumed that the amplitude increases proportionally to the square of the fibre diameter (Lorente de Nó, 1947; Håkansson, 1957).

$$
\phi_{p}^{\prime}(r, z)=\frac{a^{2} \sigma_{i} K}{4 \sigma_{e}}\{\overbrace{\frac{\alpha}{\sqrt{\left(z-s_{1}-z_{i}\right)^{2}+r^{2}}}}^{\text {Pole }}-\frac{1}{\sqrt{\left(z+s_{1}-z_{i}\right)^{2}+r^{2}}}+\frac{1-\alpha}{\sqrt{\left(z+s_{2}-z_{i}\right)^{2}+r^{2}}}\},
$$




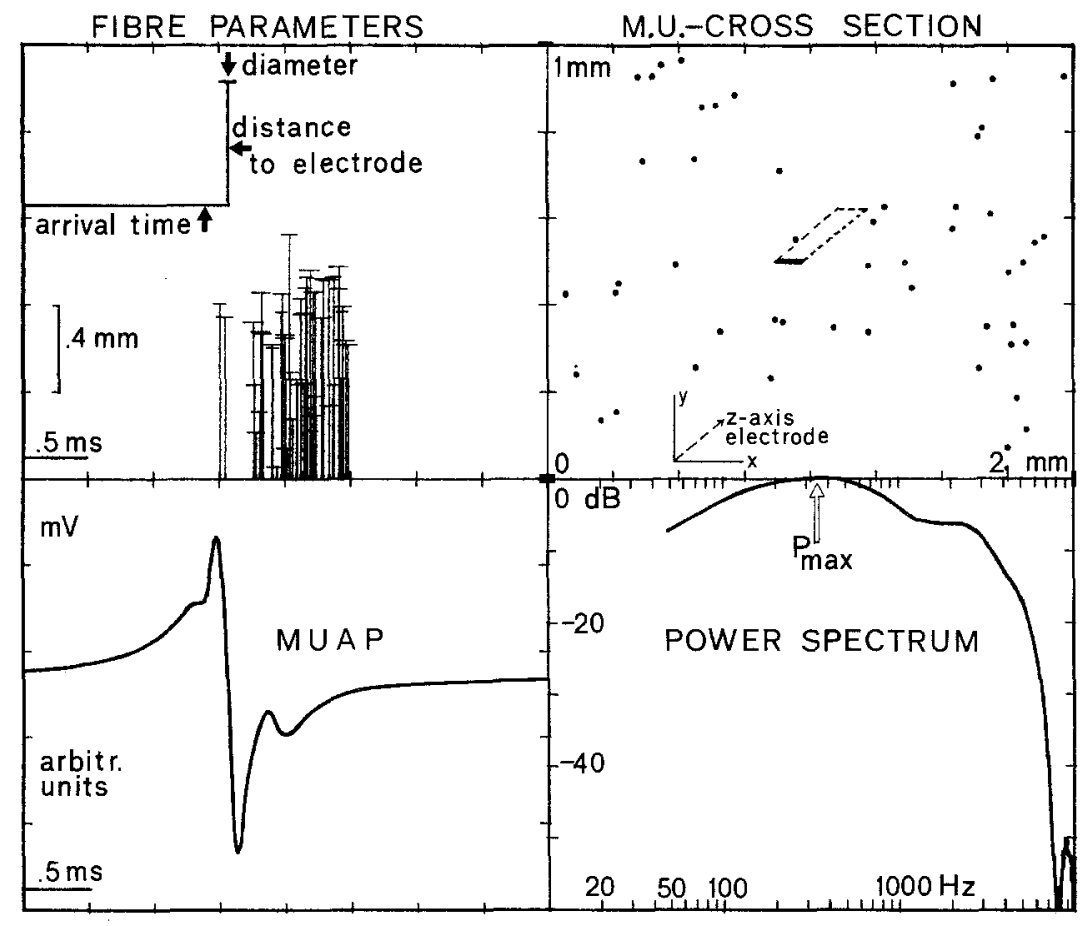

Fig. 2. Example of a computer plot of a muap simulation. Besides the muap and its power spectrum the parameters used are also drawn. In the upper left quadrant for each fibre; the dispersion of arrival time, distance to the electrode-centre and fibre diameter are presented as indicated. In order to visualize the main parameters, in the upper right quadrant a cross section is given showing the muscle fibres of the motor unit and the electrode. The horizontal line of the electrode is an exact representation of the $X$ and $Y$ coordinates of this electrode (see also Fig. 1b). The electrode was directed along the $Z$-direction which is perpendicular to the drawing. This is indicated in perspective by means of a dotted oblique line. Using these data the muap is calculated. It is presented in the lower left quadrant. Finally a normalized power spectrum of this muap is plotted in the lower right quadrant (to $\log / \mathrm{dB}$ scales). The arrow at the power spectrum indicates the low frequency maximum
In (1): $a$ represents the fibre diameter; $a$ is chosen from pdf; $r$ represents the distance from point $P$ to a muscle fibre $r^{2}=\left(x-x_{i}\right)^{2}+\left(y-y_{i}\right)^{2} ; x$ and $y$ are chosen from a pdf; $z$ represents the position of the tripole on the muscle fibre $z=z_{0}+d-v \cdot t$; the dispersion $d$ is chosen from a pdf, and $v$ represents the velocity of the action potential and $t$ is time; $\sigma_{i}$ and $\sigma_{e}$ are the conductivities in the intracellular and extracellular medium; $K$ is a constant.

The function $\phi_{p}$ for a cylindrically anisotropic medium can be easily obtained by taking:

$\phi_{p}(r, z)=3 \cdot \phi_{p}^{\prime}(r \sqrt{5}, z)\left(\right.$ Rosenfalck $\left.^{\prime} 69: \frac{\sigma_{r}}{\sigma_{z}}=1 / 5\right)$.

The tripole parameters $\left(s_{1}, s_{2}, \alpha\right)$ were assumed to be equal for each fibre. A possible relation between diameter and conduction velocity can be taken into account by adjusting the pdf of arrival times.

\subsection{Influence of Electrode Dimensions}

The potential of a not point-shaped electrode was approximated by spatial integration of the function $\phi_{p}$ with respect to the electrode surface $A$

$\phi_{\mathrm{cl}}=\frac{1}{A} \iint_{A} \phi_{p}(r, z) d A$
For the calculation of this integral it was convenient to take a rectangular lead-off area parallel to the muscle fibres. In Figure $1 b$ the position of the electrode and one muscle fibre is shown to indicate the coordinates used. The solution of the integral (3) was obtained in two steps:

Step 1: integration in the $X$-direction in an analytic way (Boon and Griep, 1976). In order to evaluate the contribution of the different poles [see Eq. (1)] the contribution of Pole 1 to the electrode potential $\phi_{\mathrm{el}}$ is considered,

$$
\begin{aligned}
& \phi_{\mathrm{el} ; \text { Pole } 1}=\frac{1}{A} \iint \phi_{p ; \text { Pole } 1}(r, z) d A \\
&=\frac{1}{A} \iint 3 \cdot \phi_{p ; \text { Pole }}^{\prime}(r \sqrt{5}, z) d A \\
&=\frac{a^{2} \sigma_{i} K}{4 \sigma_{e}} \cdot \frac{3}{A} \cdot \int_{-1 / 2 z_{1}} \underbrace{\int_{x_{1}}^{1 / 2 z_{1} x_{2}} \frac{\alpha}{\sqrt{\left(z-s_{1}-z_{i}\right)^{2}+r^{2} \cdot 5}}}_{I_{x}} d x_{i} \cdot d z_{i} .
\end{aligned}
$$

For the solution of integral $I_{x}$ see the Appendix.

Step 2: Integration in the $Z$-direction by means of a numerical convolution (Griep and Boon, 1977).

Equation (4) can be written as:

$\phi_{\mathrm{el} ; \mathrm{Pole} \mathbf{1}}(z)=\frac{a^{2} \sigma_{i} K}{4 \sigma_{e}} \cdot \frac{3}{A} \int_{-1 / 2 z_{1}}^{+1 / 2 z_{1}} I_{x}\left(z-z_{i}\right) \cdot d z_{i}$, 
a
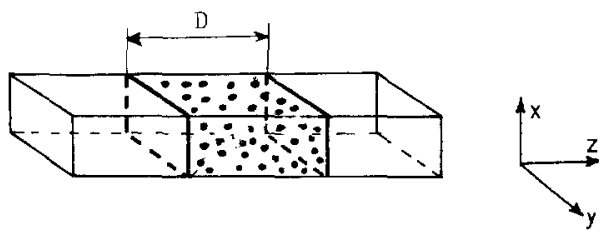

b

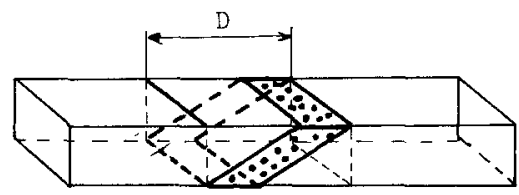

Fig. 3a and b. Distribution of arrival times. In these two figures the muscle is represented very schematically as a bar, the position of each dot represents an arrival time of a single fibre potential to the electrode. Two situations are drawn: a the arrival times are randomly spread in a rectangular zone; $\mathbf{b}$ the arrival times are randomly spread in a relatively small oblique zone (mainly due to the possible positions of the end-plates)

or in the time domain:

$$
\begin{aligned}
\phi_{\text {el; Pole } 1}(t)= & \frac{a^{2} \sigma_{i} K}{4 \sigma_{e}} \cdot \frac{3}{A} \cdot-v \int_{+1 / 2 \frac{z_{1}}{v}}^{-1 / 2 \frac{z_{1}}{v}} I_{x}\left(v t-v t_{i}\right) d t_{i} \\
= & \frac{a^{2} \sigma_{i} K}{4 \sigma_{e}} \cdot \frac{3}{A} \cdot v \int_{-\infty}^{+\infty} \\
& I_{x}\left(v t-v t_{i}\right) h\left(t_{i}\right) d t_{i} \text { with } h\left(t_{i}\right) \\
= & 1 \text { for }-\frac{z_{1}}{2 v}<t_{i}<\frac{z_{1}}{2 v}=0 \quad \text { else } \\
= & \frac{a^{2} \sigma_{i} K}{4 \sigma_{e}} \cdot \frac{3}{A} \cdot v \cdot I_{x}(y t) * h(t)
\end{aligned}
$$

Where $*$ means a convolution.

For all fibres of the motor unit with the same conduction velocity a convolution with the same impulse response has to be carried out. Therefore it is more convenient to summate the contributions of all fibres and calculate the convolution only once afterwards.

\subsection{Choice of Parameters}

The parameters used in this study all refer to the $m$. soleus of the rat. This muscle contains about 2500 muscle fibres (Close, 1967; Frank et al., 1975). It was assumed that there are approximately 30-50 motor units (own measurements). A motor unit containing 50 fibres was simulated. Considering the results of Kugelberg (1973) it seems reasonable to accept a random (rectangular) distribution for the pdf of the fibre positions. For the cross section of a motor unit territory an area $1 \times 2 \mathrm{~mm}$ was chosen. It was not possible to obtain a precise pdf of the fibre diameters. Several authors investigated the fibre diameter (Yonemura, 1967; Edström and Kugelberg, 1968).
Finally a random (rectangular) distribution between $20-40 \mu \mathrm{m}$ was chosen. Although a Gaussian distribution might be more appropriate, it can be argued that the effects of this difference are subordinate.

The pdf, describing the dispersion in arrival time can be chosen in different ways. These ways are illustrated in Fig. 3.

In Figure 3a the pdf is chosen randomly (within a certain interval) so the arrival times are independent of the fibre positions within the muscle. In Figure 3b however the pdf is chosen randomly in an oblique zone. In this way the arrival times depend also on the fibre position within the muscle. Such an pdf is in accordance with a skew situation of the end-plate zone in a muscle (see Fig. 1a). The width $D$ of the distribution function of arrival times was taken $1.25 \mathrm{~ms}$. If the dispersion is only caused by the distribution of end-plate positions, this interval relates to an upper limit of the motor end-plate region of $1.25 \times 4=5 \mathrm{~mm}$ (conduction velocity: $4 \mathrm{~m} / \mathrm{s}$ ). This region is comparable with the results of Frank et al. (1975) and Ypey (1976). Besides these authors indicate a skew end-plate region within the muscle.

At situation $3 \mathrm{~b}$ the shape of the "parallelogram function" can be indicated by a factor DCOR between 0 and 1 . In this case the arrival time for the $i^{\text {th }}$ fibre $D_{i}$ is :

$D_{i}=D\left\{\operatorname{DCOR} \frac{x_{i}}{x_{\text {terr }}}+(1-\mathrm{DCOR}) \cdot R_{i}\right\}$,

where: $x_{i}$ is the $x$-coordinate of the $i^{\text {th }}$ fibre; $x_{\text {terr }}$ is the territory dimension in the $x$-direction; $R_{i}$ is a random variable between 0 and 1 .

All the simulation results refer to a value of $\mathrm{DCOR}=0.5$, unless otherwise indicated.

The tripole parameters were adjusted on the basis of literature (Rosenfalck, 1969) and our own measurements. With a conduction velocity of $4 \mathrm{~m} / \mathrm{s}$ the value of $s_{1}=250 \mu \mathrm{m}$. The other parameters were stated on : $\alpha=0.8$ and $s_{2}=1250 \mu \mathrm{m}$. The dimensions of the electrode can be chosen arbitrary.

All the simulation results refer to unipolar electrodes, unless otherwise indicated.

\section{Results}

\subsection{Influence of Dimensions and Position of the Electrode on the Muap}

Figure 4 shows simulated muaps, "registrated" both by a point-shaped electrode and a macro electrode (dotted curves: lead-off area : $200 \times 760 \mu \mathrm{m}$ ) each for two different positions with respect to the motor unit territory. 


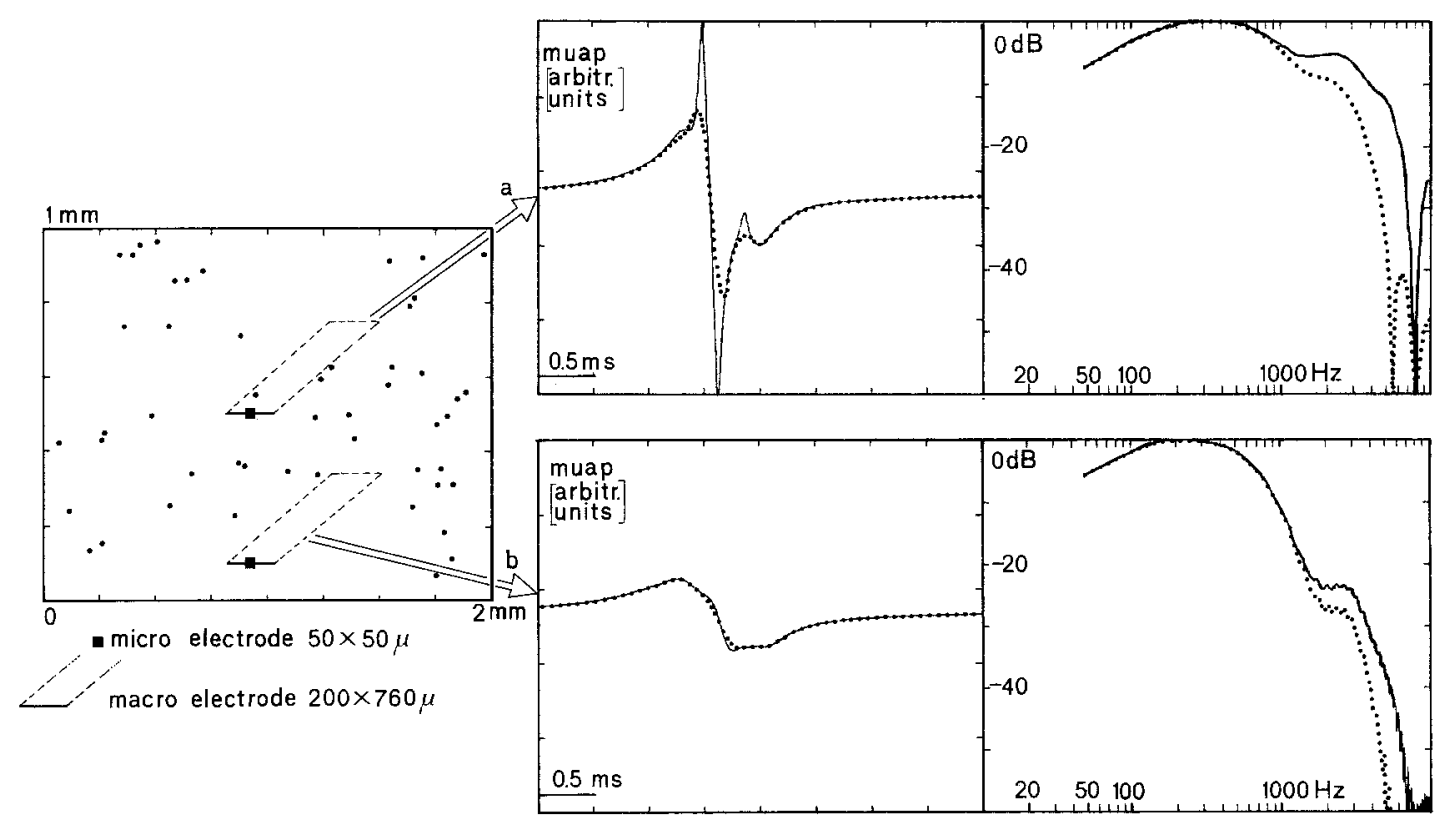

Fig. 4. Influence of the electrode dimensions and position with respect to the motor unit territory on the muap and its power spectrum. The dotted lines refer to an electrode with a lead-off area of $200 \times 760 \mu \mathrm{m}$, while the continuous lines refer to a micro electrode. In the left part the positions of the muscle fibres with respect to the electrode and the dimensions of the electrode are indicated. The change to smooth biphasic patterns, for both electrode types from intra to extraterritorial registrations is clearly shown

Within the motor unit territory (Fig. 4a) there was a noticeable difference in amplitude and slightly in shape. The actual shape of an intraterritorial observed muap is of course strongly determined by the fibres lying right next to the electrode. This dependence was most obvious for a point shaped electrode: rapid changes caused by the nearby fibres were observed. These rapid changes correspond to high frequency components in the power spectrum. A low-pass filtering effect of the macro electrode, caused by the surface integration, was obvious in intraterritorial observation (Fig. 4a). If the electrode was at the "border" of the motor unit territory there was hardly any difference between the "registrations" of the two electrodes.

Low and high frequency contents could be distinguished in the power spectra. The low frequency part was characterized by the first maximum in the spectrum (see Fig. 2).

As will be shown the amplitude of this first maximum contained general information about the motor unit. Extraterritorial simulated muaps were dominated by the low frequency content. In the time domain only a biphasic slow wave appeared.

In the intraterritorial simulated muaps a similar slow biphasic wave could also be recognized as a part of the total muap. The low frequency maximum in the spectrum showed a slight shift to lower frequencies with increasing distance of the electrode (Fig. $4 a$ and b) as can be expected considering the volume conduction theory.

\subsection{Influence of the Distribution of Motor End-plates on the Muap}

It is interesting to know if the shape of a muap depends on the arrival times of the individual single fibre potentials at the electrode.

Anatomical studies of the situation of the motor end-plates (Coërs and Woolf, 1957; Ypey, 1975; Frank et al., 1975) suggest a small oblique zone as shown in Figure $3 \mathrm{~b}$. Such a geometrical situation implies a correlation between the position of a fibre with respect to the cross section of the muscle and the arrival time. Therefore the influence of some arrival time functions, which depend on the distribution of motor end-plates on the muap was simulated.

In Figure 5a the end-plates were randomly distributed in the whole end-plate region, a polyphasic muap was obtained. If the end-plates were exactly positioned in a skew plane a biphasic muap with a higher amplitude was obtained. In this Figure $5 \mathrm{c}$ it is assumed that differences in arrival times are only caused by different end-plate positions with respect to the cross-section of the muscle $(\mathrm{DCOR}=1)$.

In Fig. 5b an "intermediate" structure $(\mathrm{DCOR}=0.3)$ was simulated: the muap is almost biphasic.

It is obvious that the structure of the motor endplane zone influences the muap strongly. 

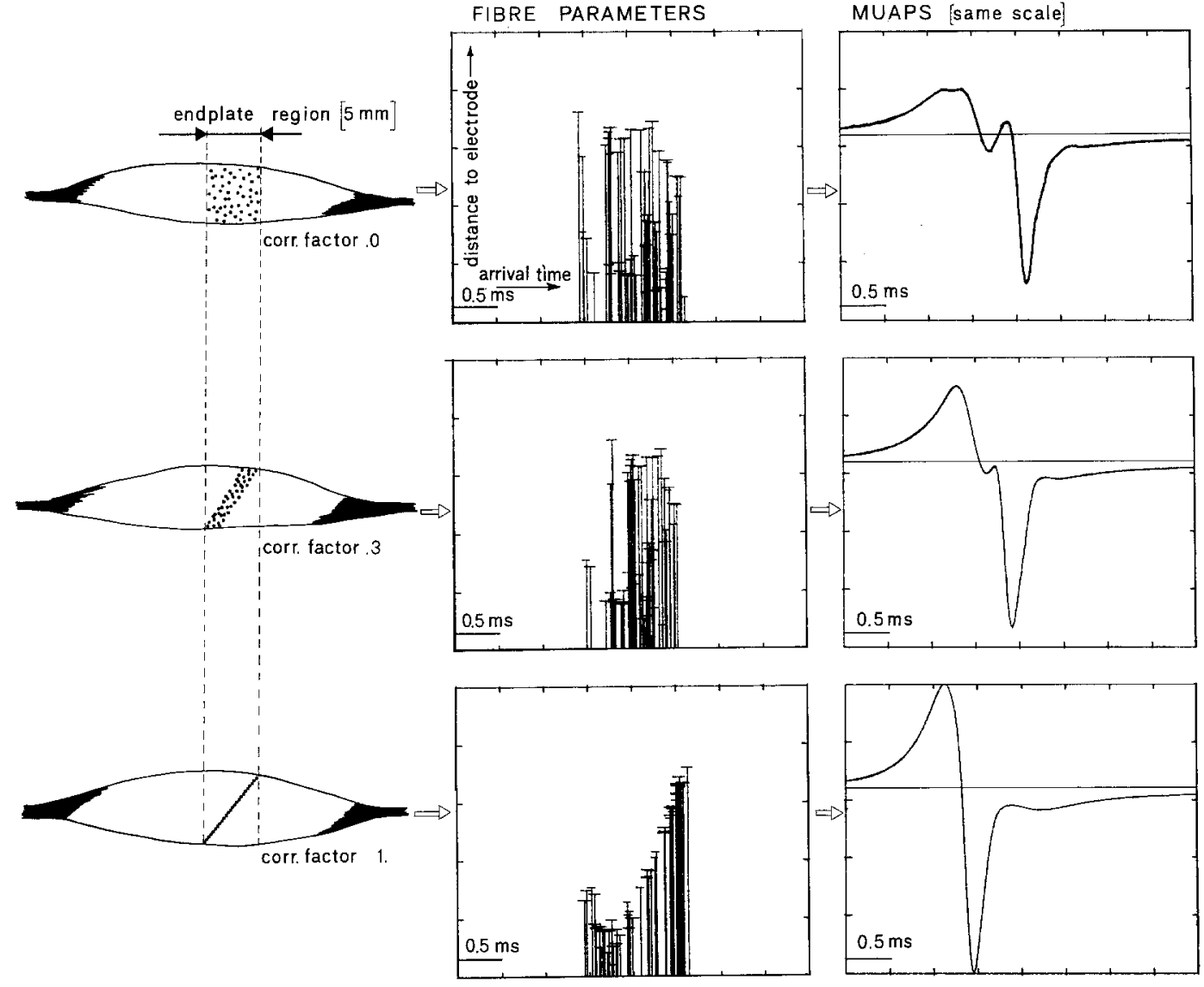

Fig. 5. The influence of the distribution of motor end-plates on the muap. From the simulation result in the top of the figure, it can be seen that with end-plates, that are randomly distributed in the whole end-plate region, a more polyphasic muap is obtained. If the end-plates are exactly positioned in a skew plane a biphasic muap with a higher amplitude is obtained. With these simulations it is assumed that differences in arrival times are only caused by different end-plate positions with respect to the cross-section of the muscle. In the middle of the figure an "intermediate" structure (DCOR $=0.3$ ) was simulated : the muap is almost biphasic. It is obvious that the structure of the motor end-plate zone influences the muap strongly

\subsection{Changing Dispersion of Arrival Times}

An enlarged dispersion is an important pathological symptom which can be observed e.g. in neuropathies (Hopf and Struppler, 1974).

Figure 6 shows an example of the influence of an enlarged dispersion on the form of the muap and its spectrum.

The electrode $(100 \times 380 \mu \mathrm{m})$ was situated in the centre of the motor unit territory. In Fig. $6 a$ the maximum difference in arrival time is $1.25 \mathrm{~ms}$. A muap which is almost biphasic is apparent. An enlargement to $2.5 \mathrm{~ms}$ (Fig. 6b) generated a more polyphasic muap. The change to a more polyphasic pattern is a wellknown phenomenon. Usually a polyphasic muap resulted in more power in the high frequency part of the power spectrum.

From Fig. 6 it can be concluded that the maximum of the low frequency part shifts to a lower frequency. In general this frequency was related to the duration of the muap and therefore also to the dispersion of the arrival times.

\subsection{Fibre Density and Motor Unit Territory}

Differences in the number of fibres of one motor unit can be caused by variations in the fibre density and/or variations in the size of the motor unit territory. Of course an increasing number of fibres will result in a higher amplitude of the muap as long as compensation, due to superposition of negative and positive phases of different fibre potentials is not complete. It can be argued that such a compensation will hardly affect the low frequency part of the spectrum.

Therefore the amplitude of the low frequency maximum is an interesting parameter. In Fig. 7 this parameter is plotted as a function of the number of fibres in an intraterritorial simulated muap. Besides this parameter also the top-top amplitude of the time registration is indicated in Fig. 7 in order to compare this with the low frequency maximum parameters.

In Fig. 7a the number of fibres is proportional to the density by taking a constant cross-section area of the motor unit, whereas in Fig. $7 \mathrm{~b}$ this number is proportional to the area, implicating a constant 

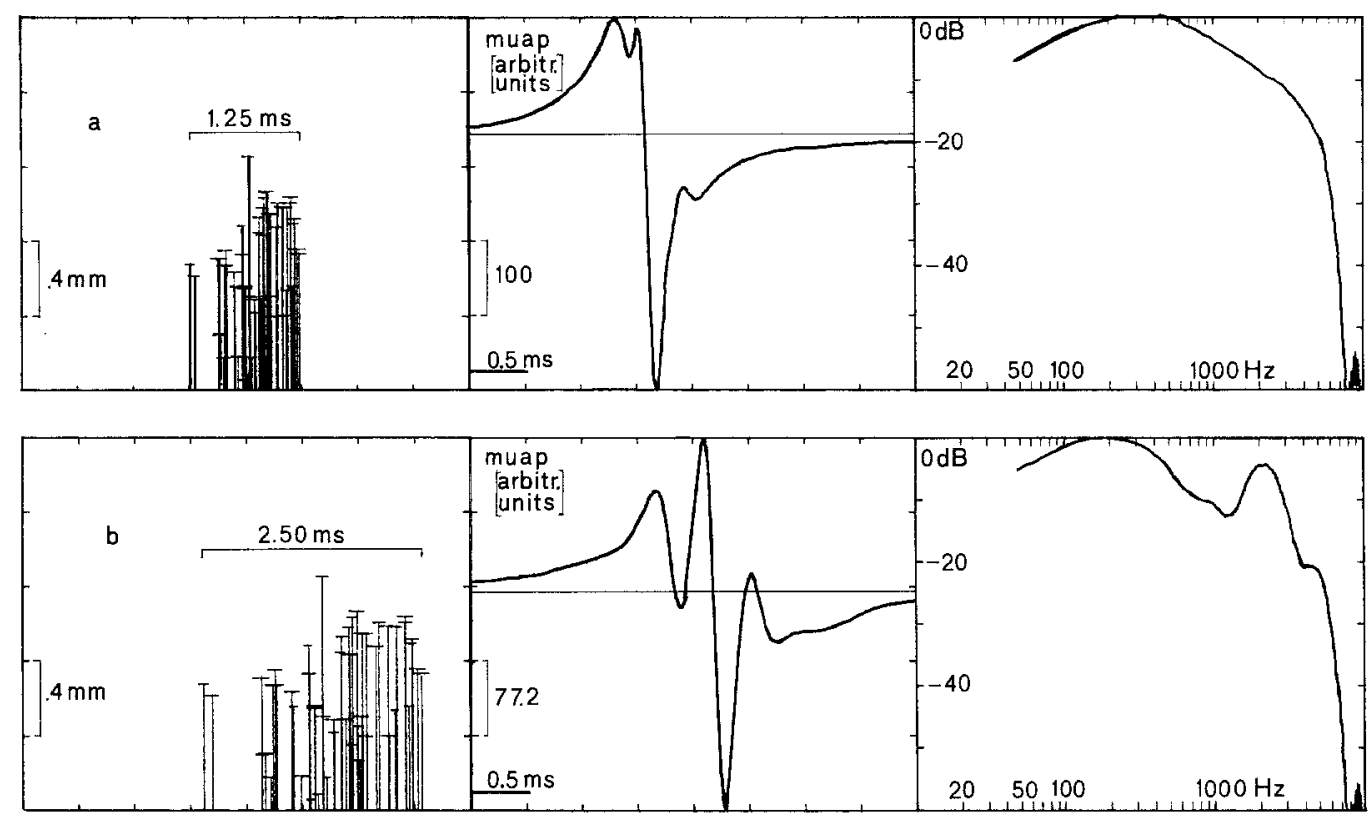

Fig. 6a and b. Influence of an enlarged dispersion of arrival times on the muap and its power spectrum. In the upper part a situation is shown, with a certain dispersion of arrival times, while in the lower part the dispersion is (proportionally) enlarged. This causes a more polyphasic pattern and usually more power in the high frequency part of the power spectrum. The maximum of the low frequency part is shifted to a lower frequency

density. In the first case the parameter $\sqrt{P_{\max }}$ shows an almost linear dependence with respect to the fibre density while the muap amplitude is rather variable.

In the lower part the fibre density is taken constant. There is a clear relation between $\sqrt{P_{\max }}$ and territory diameters.

The muap amplitude is almost independent of the territory diameter. All results are obtained by assuming the lead-off electrode to be positioned in the geometrical centre of the territory.

\subsection{Variability of Some Muap-parameters with Respect to the Electrode Position}

In order to illustrate the influence of the actual position of the electrode within a certain area of the motor unit territory we simulated 42 muaps. The electrode positions were chosen arbitrary in the following areas:

1. approximately in the geometrical centre of the motor unit, 2. at the boundary, 3. between the centre and the boundary, and 4 . outside the motor unit territory (three different regions).

From the simulation results means and standard deviations were calculated based on the muaps led off from one area, characterized by its mean distance to the geometrical centre of the motor unit.

In Fig. 8 the ratio standard deviation/mean is plotted as a function of the distance to the centre, both for the top-top amplitude as well as for the square root of the amplitude of the low frequency maximum. Apparently the latter is a less fluctuating parameter. Therefore it can be used in order to obtain information
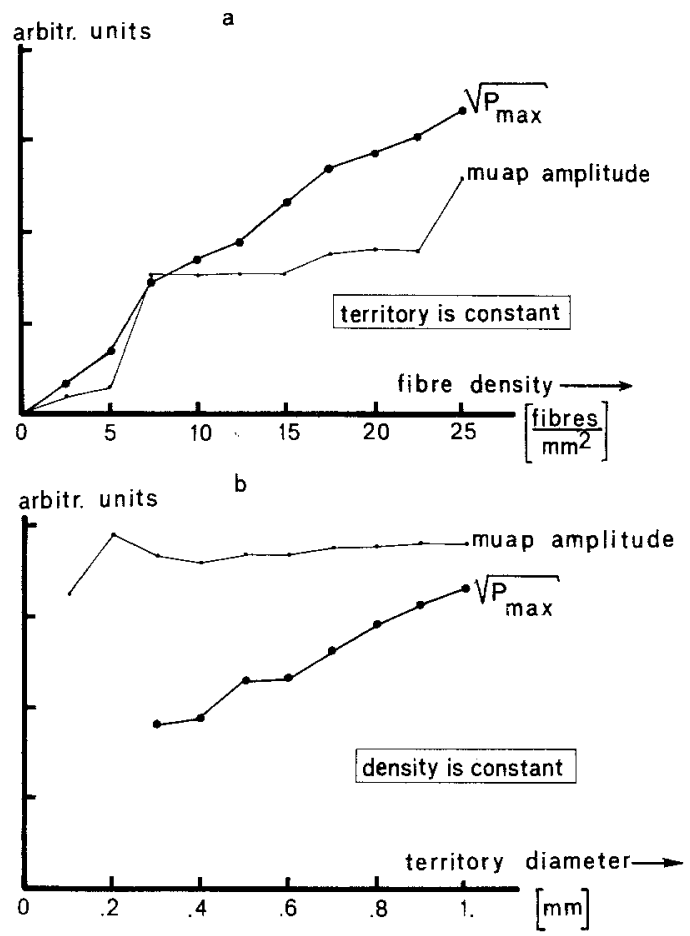

Fig. 7a and b. Relation between two muap parameters and fibre density or territory diameter. In this figure it is presented how the muap amplitude and $\sqrt{P_{\max }}$ are related to the fibre density as well as the territory diameter. In the upper part the m.u. territory is taken constant and the fibre density is taken variable. The parameter $\sqrt{P_{\max }}$ shows an almost linear dependence with respect to the fibre density while the muap amplitude is rather variable. In the lower part the fibre density is taken constant. There is a clear relation between $\sqrt{P_{\max }}$ and territory diameter. The muap amplitude is almost independent of the territory diameter. All results are obtained by assuming the lead-off electrode to be positioned in the centre of the territory 


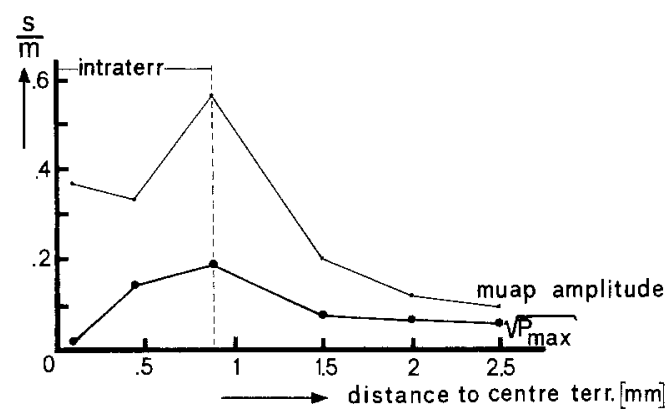

Fig. 8. The ratio standard deviation/mean $\left(\frac{s}{m}\right)$ of simulated muaps, plotted as a function of the distance from the electrode to the centre of the motor unit, indicating the variability of the parameters top-top amplitude and $\sqrt{P_{\max }}$ (electrode $100 \times 380 \mu \mathrm{m}$ ). The upper curve refers to the top-top amplitude, the lower curve refers to the square root of the low frequency maximum, which is clearly a less fluctuating parameter and therefore it is an interesting parameter for further investigations

about fibre density and/or size of the motor unit (see also Fig. 7).

\subsection{Selectivity of an Unipolar and a Bipolar Electrode}

The usefulness of the model for studying different kind of electrodes will be illustrated by a case study concerning the selectivity-characteristics of unipolar and bipolar electrodes. Two partly overlapping motor units (Nos. 1 and 2) were considered, which were supposed to be active at the same time (see Fig. 9).

The recording electrode was positioned in the centre of motor unit 1 and just outside motor unit 2 .
In the upper part of Fig. 9 it is shown how the muap of motor unit 1 simulated with an unipolar electrode, is strongly interfered with the muap originating of the second motor unit.

However if a bipolar registration (the two electrodes are positioned parallel to the muscle fibres) is simulated, with an interelectrode distance of $0.3 \mathrm{~mm}$, the electrode seems to be very selective for the activity of motor unit 1 (lower part of Fig. 9).

The powerspectra are also indicated. It can be seen that a bipolar electrode acts as a high-pass filter.

On increasing the interelectrode distance the power spectrum showed regular "dips" from which the conduction velocity of the muscle fibres can be derived (Linström, 1974).

\section{Discussion}

In the previous part results of the stochastic simulation model have been presented. It was a first attempt to study possible muaps by "imitating" the motor unit both from the anatomical as the physiological point of view. The model is strongly based on the description of the single fibre potential. If only one fibre is considered, the model is equivalent to the model of Ekstedt and Stålberg (1973) however in our model also anisotropy is taken into account. The description of the single fibre potential has its limitations especially when the muscle fibre is too close to the electrode. In this case a more correct description can be given as is shown by Rosenfalck (1969) and Dimitrov and Dimitrova (1974).

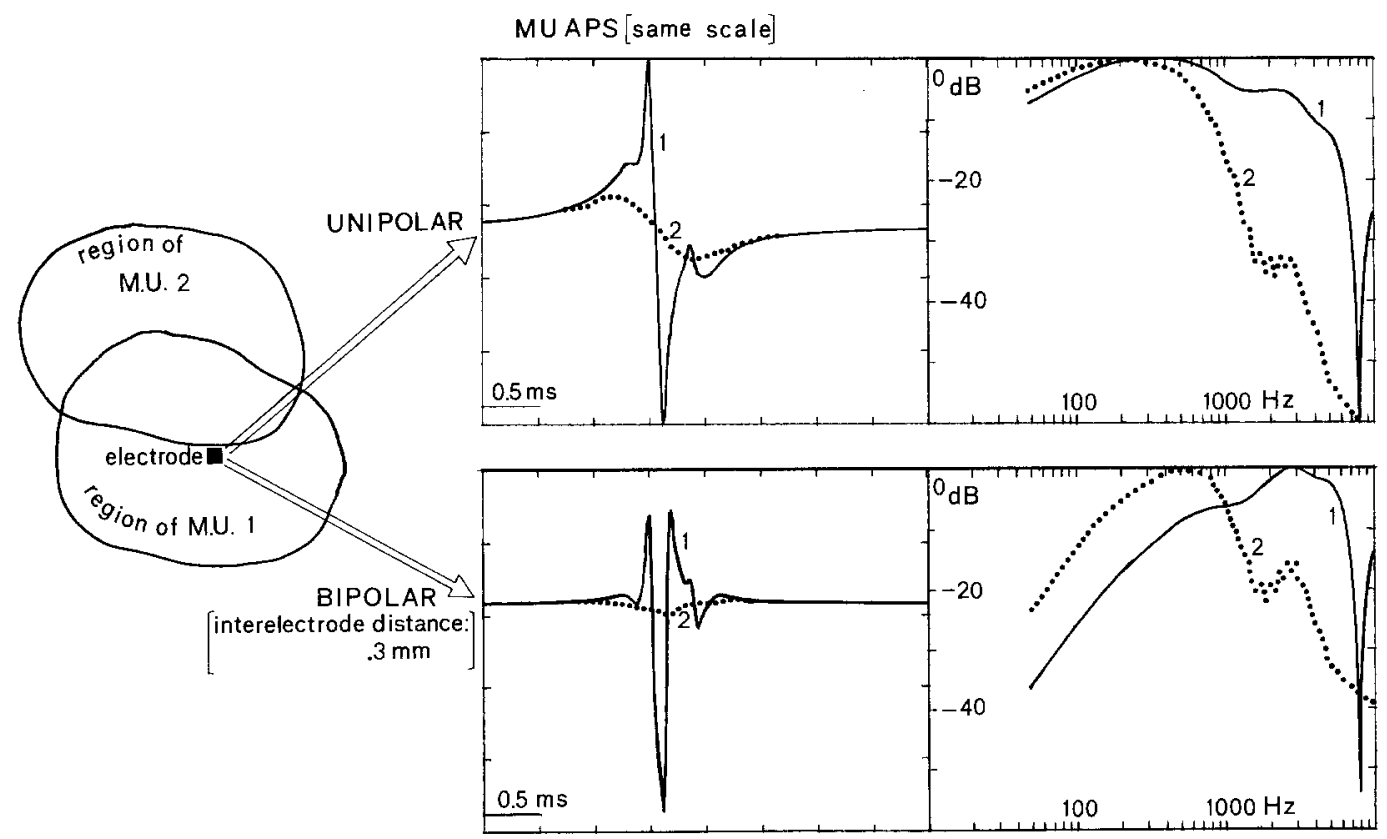

Fig. 9. Selectivity of an unipolar and bipolar electrode. Two overlapping motor units are active at the same time. If an unipolar recording is simulated, the activity of motor unit 1 is strongly interfered with the activity of motor unit 2 . However if a bipolar recording is simulated with a small interelectrode distance $(0.3 \mathrm{~mm})$ this electrode seems to be very selective for the activity of motor unit 1 ; a distorted muap is obtained. The power spectra are also indicated. It can be seen that a bipolar electrode acts as a high-pass filter 
It is of course possible to build in a more general applicable algorithm. This was not done here because then the program would be very time-consuming while improvements were expected to be marginal.

In literature a number of EMG-models concerning single muaps are mentioned (George, 1970; Lindström et al., 1976). These models have an analytical approach and the results refer to so called expected values (statistical first and second order moments). Especially the work of Lindström et al. on this subject is very extensive. They calculated expected values of the Fourier and power spectrum using the pdfs of the stochastic variables already mentioned. With the stochastic simulation model these pdfs are basically not used as a weighting function. In our model the contribution of each fibre to the muap is individually simulated using parameter-values that were chosen for each fibre separately, according to the same pdf. Only if the number of fibres in a motor unit is large enough, do the two methods lead to comparable results. For extraterritorial recorded muaps the number of fibres is usually large enough so that the expected values form a good approximation of a particular muap. For intraterritorial recorded muaps however it is the fraction of fibres in close vicinity to the electrode that is not large enough to make statistical weighting useful. Single fibre potentials that can be observed separately in reality and also which our model (see Fig. 3) are "smoothed away" by using the pdf as a weighting function.

This effect can be easily demonstrated by assuming only one fibre potential $f(t-\tau)$. Only the arrival time $\tau$ is taken as a stochastic variable. On this way the expected value is:

$E[f(t-\tau)]=\int_{-\infty}^{\infty} f(t-\tau) p(\tau) d \tau=\bar{f}(t)$,

where $p(\tau)$ represents the probability density function of the arrival time $\tau$. The shape of $\bar{f}(t)$ differs of course of the shape of $f(t)$ and it must be emphasized that always (at every experiment) the shape $f(t)$ will be measured and never $\bar{f}(t)$ !

The results of the simulation model will depend strongly on the parameters used, just as in reality. This implies that when using the model to study a specific muscle, these parameters must be investigated as well as possible. Now an extensively investigation is in progress to test the simulation model thoroughly by measuring all the parameters used in the model by electrophysiological and histochemical techniques.

From our own measurements it appeared that the variability in registrated muap-shapes is comparable with our simulation results.

In the model the dispersion of arrival times is taken statistically dependent on the position of the muscle fibre within the motor unit. From the simulation results (Fig. 5) it can be concluded that on increasing the correlation between position and arrival time, less polyphasic patterns will appear.

Physiological studies of Stålberg and Thiele (1975) by means of single fibre electromyography indicate a relatively small time interval between two fibres that are lying close to each other; this also reveals a possible correlation. Another parameter of interest is the pdf which determines the scattering of muscle fibres of a motor unit within the muscle. In the simulation model the fibres were randomly (homogeneous) spread. Although in literature these pdf's are not investigated quantitatively, data about motor unit cross sections (also of different muscles) suggest such a random distribution (Kugelberg, 1973; Burke et al., 1974; Brandstater and Lambert, 1973; Stålberg et al., 1976). In pathological cases however this distribution can change significantly.

On observing several simulated muaps it is apparent how variable the top-top amplitude of the muap can be if an intraterritorial registration is made. The variations of the distance between the closest fibres and the electrode are very dominant.

This is the reason why Gydikov (1976) only used extraterritorial muaps for studying the motor units (see also Buchthal and Rosenfalck, 1973). Stålberg (1976) argued that the intraterritorially recorded muap is only determined by the "closest fibres" already mentioned.

With respect to these findings the simulation results indicate that the low-frequency maximum of the power spectrum is an interesting parameter to describe features concerning the whole motor unit, both with extraterritorial as with intraterritorial measurements.

As could be expected it is evident from the simulation results with micro- and macro-electrodes (Fig. 4) that the micro electrode gives a signal that is mainly related to the closest single fibre(s) while the macro electrode signal (especially for larger macroelectrodes) is more related to all the fibres of the motor unit. This suggests an electrode construction for practical investigations in which a macro and micro electrode are combined. For example the micro electrode signal can be used as a trigger signal to average the macro electrode signal, in order to obtain isolated "macro-muaps" from interference patterns (Leifer and Pinelli, 1976).

Finally it is concluded that the presented simulation model is a powerful tool for investigating the basic problems: behaviour of existing and new types of electrodes and the relations between muaps and motor unit parameters. 


\section{Appendix}

Solution of integral $I_{x}$ :

$$
\begin{aligned}
I_{x} & =\int_{x_{1}}^{x_{2}} \frac{\alpha}{\sqrt{\left(z-s_{1}-z_{i}\right)^{2}+r^{2} \cdot 5}} d x_{i} \\
& =\alpha \int_{x_{1}}^{x_{2}} \frac{1}{\sqrt{\left(z-s_{1}-z_{i}\right)^{2}+5\left(x-x_{i}\right)^{2}+5\left(y-y_{i}\right)^{2}}} d x_{i} \\
& =\frac{\alpha}{\sqrt{5}} \cdot \ln \frac{\left(x-x_{1}\right)+\sqrt{\left(x-x_{1}\right)^{2}+b^{2}}}{\left(x-x_{2}\right)+\sqrt{\left(x-x_{2}\right)^{2}+b^{2}}} \text { for: } x>x_{2} \\
& =\frac{\alpha}{\sqrt{5}} \cdot \ln \frac{\left(x_{2}-x\right)+\sqrt{\left(x_{2}-x\right)^{2}+b^{2}}}{\left(x_{1}-x\right)+\sqrt{\left(x_{1}-x\right)^{2}+b^{2}}} \text { for: } x<x_{1},
\end{aligned}
$$

where: $b^{2}=\left(y-y_{i}\right)^{2}+\frac{\left(z-s_{1}-z_{i}\right)^{2}}{5}$.

For $x_{1}<x<x_{2}$ a combination of these formulas is obtained.

Acknowledgements. The authors wish to express their appreciations to Willemien Wallinga-de Jonge and Herman Boom for their suggestions and comments, Dr. F. H. Lopes da Silva for reading and Truus Steijlen for typing the manuscript.

\section{References}

Boon, K.L., Griep,P.A.M.: The motor unit action potential: study with a stochastic simulation model and development of a new mechano-electrical needle transducer. In : Proc. Isek, Arrigo, A ed. Pavia, pp. 22-26, 1976

Buchthal,F., Rosenfalck,P.: On the structure of motor units. In Desmedt,J.E. ed., New Developm. Electromyogr. clin. Neurophysiol., Vol. 1, pp. 71-85. Basel: Karger 1973

Burke, R.E., Levine,D.N., Salcman,M., Tsairis,P.: Motor units in cat soleus muscle: physiological, histochemical, and morphological characteristics. J. Physiol. 238, 503-514 (1974)

Brandstater, M.E., Lambert,E.H.: Motor unit anatomy. In: New Developm. Electromyogr. clin. Neurophysiol,, Vol. 1, pp. 14-22, Desmedt,J.E., ed. Basel: Karger 1973

Close, R.: Properties of motor units in fast and slow skeletal muscles of the rat. J. Physiol. 193, 45-55 (1967)

Coërs,C., Woolf, A.L.: The innervation of muscle, pp. 14-20. Oxford: Blackwell 1957

Dimitrov,G., Dimitrova, N. : Extracellular potential field of a single striated muscle fibre immersed in anisotropic volume conductor. Electromyogr. clin. Neurophysiol. 14, 423-436 (1974)

Edström, L., Kugelberg, E. : Histochemical composition, distribution of fibres, and fatiguability of single motor units. J. Neurol. Neurosurg. Psychiat. 31, 424-433 (1968)

Ekstedt, J., Stålberg, E. : How the size of the needle-electrode leadingoff surface influences the shape of the single muscle fibre action potential in electromyography. Comp. Progr. Biomed. 3, 204 212 (1973)
Frank, E., Jansen,J.K.S., Lømo,T., Westgaard, R.: The interaction between foreign and original motor nerves innervating the soleus muscle of rats. J. Physiol. 247, 725-743 (1975)

George, R.E.: The summation of muscle fibre action potentials. Med. Biol. Eng. 8, 357-365 (1970)

Griep,P.A.M., Boon, K.L. : Study of the motor unit action potential (muap) by means of a simulation model. Electroenceph. clin. Neurophysiol. 43-4, 610 (1977)

Gydikov, A.: The potential of the motor unit. In : The motor system : neurophysiology and muscle mechanisms. Shahani, M. ed., pp. 49-71. Amsterdam: Elsevier 1976

Håkansson, C.H. : Action potentials recorded intra- and extra cellularly from the isolated frog muscle fibre in ringer's solution and in air. Acta physiol. scand. 39, 291-318 (1957)

Hopf,H.C., Struppler,A.: Elektromyographie. Stuttgart: Thieme 1974

Kugelberg,E. : Properties of the rat hind-limb motor units. In: New Developm. Electromyogr. clin. Neurophysiol., Vol. 1, pp. 2-13. Desmedt, J.E., ed. Basel: Karger 1973

Leifer,L., Pinelli,P.: Analysis of motor units by computer aided electromyography. In: Proceedings Isek, Arrigo, A., ed. Pavia, 1976

Lindström, L.: Contributions to the interpretation of myoelectric power spectra. Doctoral Dissertation, Chalmers University of Technology, Göteborg, 1974

Lindström,L., Broman, H., Magnusson, R. : Determinants of myoelectric power spectra. In: Proceedings Isek, pp. 92-95. Arrigo, A., ed. : Pavia, 1976

Lorente de Nó, R. : A study of nerve physiology. Studies from the Rockefeller Institute for Medical Research, 1947

Rosenfalck,P.: Intra- and extracellular potential fields of active nerve and muscle fibres. Doctoral Dissertation, København: Akademisk Forlag 1969

Stålberg,E., Thiele,B.: Motor unit fibre density in the extensor digitorum communis muscles. J. Neurol. Neurosurg. Psychiat. 38, 874-880 (1975)

Stålberg, E.: Single fibre electromygraphy for motor unit study in man. In: The motor system: neurophysiology and muscle mechanisms, pp. 79-92. Shahani, M., ed. Amsterdam: Elsevier 1976

Stålberg,E., Schwartz,M.S., Thiele,B., Schiller,H.H.: The normal motor unit in man. J. Neurol. Sci. 27, 291-301 (1976)

Yonemura, K.: Resting and action potentials in red and white muscles of the rat. Jap. J. Physiol. 17, 708-719 (1967)

Ypey,D.L.: Effects of muscle length and stimulus frequency on neuromuscular transmission in the frog. Doctoral Dissertation, Leiden University, 1975

Ypey,D.L. : Personal communications 1976

Received: May 13, 1978

P. A. M. Griep

Department of Electrical Engineering

Twente University of Technology

P.O. Box 217

7500 AE Enschede, The Netherlands 